\title{
Journée du 12 novembre au Centre Georges
}

Pompidou

Marie-Thérèse Jones-Davies (éd.)

\section{(2) OpenEdition}

\section{Journals}

\section{Édition électronique}

URL : http://journals.openedition.org/shakespeare/1110

DOI : 10.4000/shakespeare. 1110

ISSN : 2271-6424

\section{Éditeur}

Société Française Shakespeare

\section{Édition imprimée}

Date de publication : 1 novembre 1980

Pagination : 5-9

Référence électronique

"Journée du 12 novembre au Centre Georges Pompidou », Actes des congrès de la Société française Shakespeare [En ligne], 1 | 1980, mis en ligne le 01 novembre 2007, consulté le 26 avril 2019. URL : http://journals.openedition.org/shakespeare/1110; DOI : 10.4000/shakespeare.1110 


\section{SOCIÉTÉ FRANÇAISE SHAKESPEARE}

\section{ACTES DU CONGRĖS 1979}

DiRecteur DE LA PUblication

M.T. Jones - Davies 


\title{
COMPTE RENDU DES JOURNEES SHAKESPEARE
}

\author{
Paris, 12 - 18 Novembre 1979
}

\section{AU CENTRE GEORGES POMPIDOU}

\section{Le 12 Novembre :}

\section{Les Pièges de la Force et de la Pitié}

Cette première journée regroupait des débats et des illustrations scéniques consacrés aux pièces romaines de Shakespeare. Le choix avait été dicté par l'actualité théâtrale, puisque Guy Rétoré venait de monter Jules César au T.E.P., Roger Planchon Antoine et Cléopâtre à Villeurbanne et à Paris, et Gabriel Garran venait de présenter à Avignon et au théâtre de la Commune, Coriolan - une pièce qu'il avait déjà montée, dans un esprit fort différent, en juin 1964. D'autre part, Terry Hands avait également proposé au public parisien, au début de l'été, un Coriolan joué antérieurement en Angleterre.

\section{Jules César}

C'est de manière très libre et comme improvisée que Guy Rétoré ouvrit la journée en commentant sa mise en scène. Le décor d'André Acquart, généralement bien jugé, a suscité quelques questions : pourquoi l'abstraction ? Pourquoi dans les éléments du décor si peu d'élévation ? C'est qu'il s'agissait de figurer les ruines d'une société. Les costumes et la musique - dans ce drame qui est, selon Rétoré, celui du cheminement d'une machine politique qui finira par restaurer la tyrannie, ont été adaptés à cette option : le manteau pourpre de César symbolise le pouvoir, l'armée d'Antoine et d'Octave représente l'ordre - celle de Brutus et de Cassius signifie, visuellement, l'horreur de la guerre, la décomposition. La musique - électroacoustique - permet d'esquiver l'anecdote et le bruitage réaliste. Car les bruits et les sons devaient avoir une double signification : ce qu'entendent les conjurés en leur for intérieur et ce qu'entend Rome tout entière.

L'option de Guy Rétoré est résolument politique : il insiste, sans être démenti, sur la versatilité de la foule et le cynisme d'Antoine. L'idéalisme de Brutus - qu'il juge sévèrement - suscite un long débat, qui est peut-être celui de la pièce elle-même - sur les intentions de ce personnage, et la manière 
dont il convient de juger son échec : est-ce :un «imbécile politique », facilement manœuvré par les autres conjurés qui ont besoin de lui comme caution morale et politique ? Pouvait-il agir autrement sans ressembler à ceux qu'il combat ? Pourquoi prendre le risque d'une action politique violente si c'est pour changer les hommes et non le régime ? La conspiration n'est-elle pas trop exclusivement patricienne pour comporter des «leçons 》 recevables par les spectateurs d'aujourd'hui ? Un débat s'engage également sur les aspects surnaturels de la pièce :le positivisme de Guy Rétoré suscite quelques critiques, des interrogations sur la mise en scène, le sens de la pièce et la possibilité, aujourd'hui, de faire passer à la scène un système de croyances qui a perdu de sa force. L'éternel paradoxe d'une cuvre qui ne peut s'abstraire de l'histoire - histoire romaine, histoire élisabéthaine - mais qui fonctionne pourtant comme si elle était transhistorique est ainsi une nouvelle fois posé.

\section{Antoine et Cléopâtre}

La mise en scène de Roger Planchon fit l'objet d'une communication de Michel Bataillon, illustrée de diapositives.

Le dyptique shakespearien présenté au cours de la saison 78 par le T.N.P. réunit et oppose une œuvre écrite à la fin de la grande période tragique : Antoine et Cléopâtre, et| une rêverie romanesque inaugurant une nouvelle expérience d'écriture théâtrale : Périclès. La version scénique adoptée pour Antoine et Cléopâtre est celle d'un poète, Henri Thomas. Devant la nécessité de faire des coupes, Planchon réduit la peinture du monde de l'Orient, des combats en Asie mineure et privilégie la description concrète des mœurs militaires. Les costumes, d'inspiration moderne, présentent divers traits des sociétés militaires de ces cinquante dernières années, des uniformes iraniens contemporains aux tenues d'été de l'armée française. Les costumes civils rappellent ceux des diplomates des années 30. La description détaillée des mécanismes et rouages d'une société militaire - point focal de cette mise en scène - est enchâssée dans une autre diction qui serait le tournage de cette histoire dans un studio de cinéma. Notre mémoire de l'Egypte ancienne, explique M. Bataillon, est cinématographique. Hollywood et Cinecitta ont popularisé une représentation mentale de l'Orient qui marque notre imaginaire. Antoine et Cléopâtre fut d'ailleurs à son époque une machine spectaculaire comparable aux grands films d'aujourd'hui. Par le décalage qu'il introduit, le studio permet aussi de ne pas supprimer les scènes de bataille, coupées d'habitude et montées ici avec leur charge de réalité et leur aura d'irréalité comme de vraies-fausses-batailles reconstituées pour les besoins d'une superproduction. Dans cette fiction cinématographique, les amours des Grands et les décisions politiques majeures se nouent publiquement, devant les média. Dernière raison, essentielle : le fascisme italien, archétype des Sociétés militaires contemporaines est précisément l'époque des immenses reconstitutions cinématographiques de l'Antiquité. Le souvenir de la Rome des Césars est un des éléments dans lesquels il n'a cessé de se ressourcer. De sorte qu'entre la première rêverie théâtrale d'un Orient magique faite par un occidental et les derniers avatars occidentaux de ce rêve -- les peplums hollywoodiens ou italiens - se tisse une trame dont le studio permet poétiquement de rendre compte. Le metteur en scène de cette superproduction est le devin de Shakes- 
peare qui, seul à connaître la totalité du scénario, sait et dit le destin futur des personnages de la pièce. S'il est vrai que Shakespeare a choisi un sujet antique pour parler plus librement de son temps, Planchon s'efforce de maintenir l'éloignement en accentuant la marginalité du personnage de Cléopâtre. Plus que la souveraine empreinte de majesté et de noblesse l'actrice choisie représente d'abord la gipsy, l'étrangère, la métèque. Le retournement final par lequel elle acquiert grandeur et dignité $n$ 'en prend que plus de relief.

\section{Coriolan}

Cette œuvre fut traitée de manière complexe et variée : les divers éléments du débat avaient fait l'objet de nombreuses discussions entre Gabriel Garran et Richard Marienstras, sur un projet de ce dernier. L'intention était de montrer au public la diversité - la polarité - des interprétations que la pièce avait reçues : anti-républicaine et républicaine, brechtienne et psychologique, centrée sur un héros ou centrée sur le peuple...

Des moyens variés furent utilisés : exposés, débats, illustrations scéniques, diapositives...

Henri Fluchère fit une présentation générale de la pièce :

Coriolan est une pièce fascinante. Depuis que j'en ai entrepris la traduction et la présentation pour une édition bilingue, je n'ai cessé de me poser des questions. Je ne suis pas le seul d'ailleurs, puisque tous les commentateurs que j'ai dû consulter en présentent des lectures et des interprétations différentes. Ce qui ne manque pas d'être à la fois un enrichissement et une source de perplexités.

En effet, cette pièce romaine - la quatrième dans la série de la latinité pose à la fois des problèmes d'interprétation générale, et de caractérologie. On me dira qu'il en va de même de toutes les pièces de Shakespeare. Pas exactement. Pour Measure for Measure, par exemple, c'est le problème de la pourriture d'une société par son abandon aux vices - donc un problème moral, et le Duc qui prétend la guérir, n'est pas un personnage, mais un médiateur, un instrument. Peu importe qu'il soit atrabilaire ou divisé. Pour Hamlet, d'autre part, ce n'est pas la corruption du royaume, également « pourri », qui compte, ce sont les problèmes qui se posent sur le plan le plus personnel qui soit. Si l'on fait de Hamlet (comme le font les freudiens) un archétype de la tribu des paranoïaques ou des complexés cedipiens, se pose le problème du personnage - mais quelle que soit la réponse donnée, cela n'influence en rien la moralité du royaume. On pourrait prendre bien d'autres exemples dans le théâtre de Shakespeare.

Mais avec Coriolan, il en va tout autrement. Il y a d'abord un problème de résurrection historique. La pièce se situe à une époque de l'histoire de Rome sur laquelle les spécialistes ont des connaissances et des idées. La Rome offerte dans Coriolan est-elle une Rome romaine ou élisabéthaine ? Quel travesti de la Rome connue et de la Rome de Plutarque Shakespeare nous donne-t-il - et suivant notre réponse, sans doute en découlera-t-il une opinion sur le pouvoir imaginatif ou l'honnêteté intellectuelle du dramaturge. Mais c'est surtout un problème politique qui se pose avec insistance, problème intimement lié à celui que le comportement du personnage central pose d'un bout à l'autre de l'action. Donc personnage et situation contribuent à donner à la pièce le contexte le plus politique qui soit. On pourrait même dire que le personnage 
prend vie en fonction de la situation politique à laquelle il est confronté. Ce sont les rapports de Coriolan avec la société romaine telle que Shakespeare (après Plutarque) la conçoit, qui nous forcent à nous faire une opinion sur le personnage. Le conflit est d'ordre politique, ce qui a conduit tant de commentateurs à interpréter la tragédie en fonction de leurs préférences idéologiques, qu'elles soient d'ordre intellectuel, ouactualisées par la situation contemporaine. L'affrontement est entre celui qui aspire au pouvoir politique ou qui le détient, et ceux - le plus grand nombre - qui auront à le subir. Ainsi, suivant que le gouvernant accepte, pour gouverner, sous une forme ou sous une autre, la participation du plus grand nombre ou non, l'image donnée du fonctionnement de l'état sera tyrannique ou démocratique.

On le sait : la libido dominandi est la plus virulente des passions. Qui a le pouvoir veut le garder, qui ne l'a pas veut le conquérir. Ainsi se déchaînent les luttes pour le gouvernement de la Cité. Et les auxiliaires de ces passions : mensonge, hypocrisie, haine, cruauté, courage aussi; il faut bien le dire, rivalisent à qui mieux mieux dans leur rôle ancillaire. On donne à Machiavel un rôle en or dans les stratégies enveloppantes dont les succès sont souvent précaires et toujours contestés. Ainsi Coriolan, la plèbe et les tribuns, se livrent une lutte à mort. Et Coriolan, qui n'a que le courage, se livre en victime à la fureur de ses amis, et de ses ennemis. Mais qui est Coriolan nous intéresse autant que l'issue du conflit que nous connaissons d'avance. Car ses «idées 》 politiques sommaires sont desservies par une passion qui les dépasse. Est-il un héros orgueilleux et atrabilaire qui a mal tourné ? un «fasciste » qui n'a pas réussi ? un fils œdipien sans père (comme Hamlet) dont le cordon ombilical n'a pas été coupé avec sa mère, Jocaste, implacable triomphaliste, pour qui la mort du fils aliéné est préférable à sa survie indépendante ?...

... Ce sont là quelques-unes - quelques-unes seulement - des questions que l'on peut se poser au sujet de Coriolan. Elles apporteront des réponses au gré de la sagesse des temps, et de la perspicacité des commentateurs.

Richard Marienstras évoqua les fameuses représentations de Coriolan à la Comédie Française en 1934 : après un tableau de la situation politique en France, illustré au moyen de nombreuses photos de l'époque (agitation politique, chômage, queues devant les soupes populaires, ligues d'extrême-droite, dét1lès syndicaux...), il commenta la traduction délibérément orientée de René-Louis Piachaud et donna un aperçu du spectacle grâce aux clichés fournis par la bibliothèque de la Comédie Française. Des extraits de la presse de l'époque permirent d'évoquer les manifestations anti-républicaines qui se déroulèrent dans la salle, ainsi que les réactions du gouvernement. L'analyse de quelques scènes et de quelques clichés du spectacle montra le gigantisme inutile des moyens mis en œuvre, l'apparente efficacité des acteurs remarquablement ty pés et le caractère assez conventionnel de la mise en scène.

La partie la plus originale et la plus inattendue de ce débat sur Coriolan fut la "mise en scène » par Gabriel Garran - dirigeant trois comédiens - de la fame use analyse dialoguée de Brecht sur l'acte $I$, scène 1 . Le texte de Brecht avait été quelque peu abrégé, et interrompu ça et là par l'insertion d'extraits pertinents de la pièce. Garran utilisa également l'enregistrement de plusieurs passages de son Coriolan d'Aubervilliers afin de fournir quelques plages sonores; il utilisa aussi des projections fixes. Le spectacle prit une vie intense et sur- 
prenante - il avait nécessité des heures de mise au point -.. et le texte de Brecht se réveilla littéralement : les options brechtiennes - certaines étaient d'autant plus frappantes qu'on pouvait ainsi les évaluer avec une distance critique et la finesse de son analyse firent une profonde impression. Le but de cette " mise en scène " n'était pas seulement de faire connaître un texte à la fois trop connu et méconnu et d'insister sur l'une des lectures possibles de Coriolan : mais aussi de "dramatiser 》 la critique et le débat, de montrer sur le vif comment se déroulait le premier stade d'un travail scénique moderne, de faire sentir comment un metteur en scène peut utiliser le 《sous-texte » d'une œuvre. Dans son âpre simplicité, le dialogue de Brecht mettait à jour les tensions et les incertitudes qui, dans le cadre d'une option particulière, surgissent immanquablement dès que l'on aborde Shakespeare. En d'autres termes : quand bien même l'on accepte un penchant idéologique quelconque pour saisir la pièce, les problèmes d'interprétation et de mise en scène qu'elle pose restent aussi nombreux, aussi variés, aussi redoutables que si on l'abordait "naïvement $»-$ en ne se laissant guider que par son texte.

Terry Hands parla ensuite, avec beaucoup de brio, de sa mise en scène de Coriolan. Il illustra sa description de quelques diapositives en couleur pour souligner comment il avait cherché à créer des "tableaux » scéniques à tel ou tel autre moment de la représentation. La préoccupation de Terry Hands n'était pas, au premier abord, politique. Il insista sur les contraintes que le texte anglais de Shakespeare impose à qui le dit. Par opposition aux partis pris de la Comédie Française et à ceux de Brecht, où une part des plus maigres est faite à la psychologie, Terry Hands a fait ressortir le drame de la division civile et ses répercussions sur les personnages. La pièce, selon lui, ne serait ni de droite ni de gauche : elle a pour thème des déchirements, des ruptures, des quêtes d'amour inassouvies.

Le débat final, qui devait, sur le vif, prolonger le débat «scénique »de Brecht et s'opposer à lui, fut particulièrement animé et surprenant. Gabriel Garran parla de sa première production de Coriolan (1964) : c'était une production "brechtienne 》, où le peuple était idéalisé, où le personnage central était diminué afin de ne pas apparaître comme un "héros ». Grande différence avec sa nouvelle mise en scène : 1'hérö̈sme de Coriolan et d'Aufidius y est souligné, leur relation d'amour homosexuel et de haine y est nettement marqué. J.M. Déprats apporta de nombreuses précisions sur cette dernière mise en scène, qu'il avait suivie et étudiée. On s'interrogea sur l'éclipse relative des conceptions brechtiennes. Bernard Dort apporta un élément passionnel et passionné à la discussion : il dressa un constat de la situation actuelle de la gauche, souligna la désorientation idéologique de toute la société française : par rapport au théâtre, il en résultait un " égarement », une cécité devant l'histoire dont on pouvait se demander, ajouta R.Marienstras, si elle résultait d'un défaut de vision ou du constat de son opacité. 